\title{
Psychological distress in relation to site specific cancer mortality: pooling of unpublished data from 16 prospective cohort studies
}

\author{
G David Batty, ${ }^{1}$ Tom C Russ, ${ }^{2,3}$ Emmanuel Stamatakis, ${ }^{4}$ Mika Kivimäki ${ }^{1}$
}

Department of Epidemiology and Public Health, University

College, London, UK

${ }^{2}$ Centre for Cognitive Ageing and Cognitive Epidemiology, University of Edinburgh,

Edinburgh, UK

${ }^{3}$ Division of Psychiatry, Centre

for Clinical Brain Sciences,

University of Edinburgh,

Edinburgh, UK

${ }^{4}$ Charles Perkins Centre, Faculty of Health Sciences, University of

Sydney, Sydney, Australia

Correspondence to: D Batty

david.batty@ucl.ac.uk

Cite this as: BMJ 2017;356:j108 http://dx.doi.org/10.1136/bmj.j108

Accepted: 23 December 2016

\author{
ABSTRACT \\ OBJECTIVE \\ To examine the role of psychological distress (anxiety \\ and depression) as a potential predictor of site specific \\ cancer mortality.
}

DESIGN

Pooling of individual participant data from 16 prospective cohort studies initiated 1994-2008.

SETTING

Nationally representative samples drawn from the health survey for England (13 studies) and the Scottish health survey (three studies).

\section{PARTICIPANTS}

163363 men and women aged 16 or older at study induction, who were initially free of a cancer diagnosis, provided self reported psychological distress scores (based on the general health questionnaire, GHQ-12) and consented to health record linkage.

\section{MAIN OUTCOME MEASURE}

Vital status records used to ascertain death from 16 site specific malignancies; the three Scottish studies also had information on cancer registration (incidence).

\section{RESULTS}

The studies collectively contributed an average of 9.5 years of mortality surveillance during which there were 16267 deaths (4353 from cancer). After adjustment for age, sex, education, socioeconomic status, body mass index (BMI), and smoking and alcohol intake, and with reverse causality (by left censoring) and missing data (by imputation) taken into account, relative to people in the least distressed group (GHQ-12 score 0-6), death rates in the most distressed group (score 7-12) were consistently raised for cancer of all sites combined (multivariable adjusted hazard ratio 1.32, 95\% confidence interval 1.18 to 1.48 ) and cancers not related to smoking $(1.45,1.23$ to 1.71$)$, as well as

\section{WHAT IS ALREADY KNOWN ON THIS TOPIC}

While psychological distress (symptoms of depression and anxiety) is related to increased rates of cardiovascular disease, links with different presentations of cancer are unclear and, for selected malignancies, untested

\section{WHAT THIS STUDY ADDS}

A pooled analysis of unpublished raw data from 16 prospective cohort studies suggests associations between distress and cancer, most notably for carcinoma of the colorectum, prostate, pancreas, and oesophagus and for leukaemia

This adds to the growing evidence that psychological distress could have some predictive capacity for certain somatic diseases

With extant evidence being exclusively based on observational studies, further research is now required to clarify the extent to which each of the associations between distress and cancer is likely to be causal. carcinoma of the colorectum $(1.84,1.21$ to 2.78$)$, prostate $(2.42,1.29$ to 4.54$)$, pancreas $(2.76,1.47$ to 5.19), oesophagus $(2.59,1.34$ to 5.00$)$, and for leukaemia (3.86, 1.42 to 10.5). Stepwise associations across the full range of distress scores were observed for colorectal and prostate cancer.

\section{CONCLUSION}

This study contributes to the growing evidence that psychological distress might have some predictive capacity for selected cancer presentations, in addition to other somatic diseases.

\section{Introduction}

Although the notion of a link between mental health and physical health was first advanced centuries ago, ${ }^{1}$ the discovery of pathogenic causes for many diseases led to an extended period of quiescence in this field. In recent decades, most research has been conducted in the context of cardiovascular disease, with growing evidence implicating the psychological factors of psychosocial stress, ${ }^{2}$ cognitive function, ${ }^{3}$ and selected personality types (particularly neuroticism and conscientiousness) ${ }^{4}$ as potentially having roles at various stages of the disease process, including acting as predictive factors, markers of undiagnosed pathology, triggers of clinical events in individuals with subclinical disease, or a consequence of diagnosed somatic disease. ${ }^{5}$

The predictive capacity of a further psychological factor-psychological distress (symptoms of depression and anxiety)-in the development of cardiovascular disease has also been explored, with meta-analyses showing positive relations with risk of coronary heart disease $^{6}$ and stroke. ${ }^{78}$ Like cardiovascular disease, cancer is a major cause of death and morbidity, ${ }^{9}$ yet few studies have examined its links with distress. Various mechanisms have been implicated in linking psychological distress with cancer. Recurrent exposure to emotional distress could diminish natural killer cell function, which has been implicated in tumour cell control. ${ }^{10}$ Of particular relevance to hormone related cancers is the suggestion that symptoms of depression could lead to dysregulation of the hypothalamic pituitary adrenal (HPA) axis, increase cortisol concentrations and immunological and inflammatory responses, and inhibit DNA repair, so unfavourably impacting on multiple cancer defence processes. ${ }^{11}$ With there also being evidence that, relative to their non-distressed counterparts, people with distress symptoms are more likely to smoke, be sedentary, have an unfavourable diet, and become obese, distress could also increase the likelihood of cancer indirectly through these lifestyle related risk factors. ${ }^{12}$

The few existing prospective cohort studies, which provide the best test of an association in observational 
epidemiology, are generally small in size and show highly discordant findings with positive, null, and even inverse associations between distress and cancer reported. ${ }^{13}$ Other major gaps in understanding include the extent to which associations might be dependent on site-as cancer is not a single disease entity-and whether any apparent gradient could be generated by reverse causality-that is, distress might be a consequence of the early stages of the malignancy rather than a potential predictor. It is also the case that some studies are insufficiently well characterised to explore alternative explanations for the observed associations, including confounding by health behaviours, socioeconomic status, and systemic inflammation.

In view of the limitations of the existing evidence base, we pooled unpublished individual participant data from 16 community based prospective cohort studies that used the same methods to ascertain psychological distress, covariates, and cancer. In contrast to the more common study level meta-analytical technique, ${ }^{13}$ the use of unpublished raw data across a series of studies provides more precise estimates of the associations between uniformly defined risk markers and disease; a consistent approach to statistical control for plausible covariates and subgroup analyses; and a method that is less likely to suffer from publication bias, which besets modern epidemiology. While individual participant meta-analysis has been extensively applied to the study of the role of physiological factors for risk of disease, ${ }^{14} 15$ to the best of our knowledge, this is the first pooling of individual participant data on psychological distress, as opposed to major depression, ${ }^{13}$ and the risk of specific malignancies. In view of some of the described mechanisms potentially linking distress with selected malignancies, we hypothesised positive associations between distress and cancer for hormone related (breast, prostate, ovary) and lifestyle related (lung, colorectal, pancreas, oesophagus, stomach) cancers.

\section{Methods}

Participants were taken from the health survey for England (HSE) ${ }^{16}$ and Scottish health surveys(SHS), ${ }^{17}$ a series of geographically representative health examinations of people from the general population. Between 1994 and 2008, 16 independent, cross sectional, and methodologically near identical studies were conducted on either an annual (HSE; $n=13$ ) or occasional basis (SHS; $n=3$ ). The original purpose of these studies was to monitor secular trends in health and related behaviours. A total of 199504 men and women, aged 16-107 at baseline, were surveyed, with consenting study members linked to national health registers for vital status and, in the case of the SHS only, incidence of cancer.

\section{Measurement of psychological distress}

During a household visit, interviewers administered computer assisted personal interviewing modules that included the 12 item version of the general health questionnaire (GHQ-12). ${ }^{18}$ A widely used measure of psychological distress in population studies, the GHQ-12 comprises items capturing symptoms of depression and anxiety over the previous four weeks. Item response is based on a 4 point scale that signals the presence of a symptom ("not at all"/"same as usual" were give a score of 0; "more than usual"/"much more than usual," a score of 1). Consistent with our previous analyses, ${ }^{819-21}$ we divided people into four groups: asymptomatic (score 0), subclinically symptomatic (1-3), symptomatic (4-6), and highly symptomatic (7-12). The GHQ-12 has been validated against standardised psychiatric interviews. ${ }^{2223}$

Measurement of cancer at baseline and covariate data Cancer at baseline was based on self report (HSE), or self report and cancer registration (SHS). The validity of self reported cancer data has been validated against standard records from cancer registries. Although there is evidence that increased age and lower socioeconomic status are associated with lower levels of agreement, ${ }^{24}$ the ability of people to report a past diagnosis of cancer accurately seems to be sufficiently high for specific cancer sites of relevance to our study, such as breast, prostate, lung, and colon. ${ }^{25}$ Height and weight were measured directly, and body mass index (BMI) computed. A BMI of $\geq 30$ was used to denote obesity. ${ }^{26}$ The following characteristics were self reported: age on leaving full time education (minimum allowable age for leaving secondary school was 12-16 depending on epoch), smoking status (not a current smoker; or $<5$, 5-10, 10-15, 15-20, and $>20$ cigarettes/day), frequency of alcohol consumption (never drinker, ex-drinker, 1-2 drinks a month, 1-4 drinks a week, or $\geq 5$ drinks a week), ${ }^{1617}$ and physical activity (five or more occasions of moderate to vigorous physical activity a week).

Other covariates were collected only in certain survey years. Area-based socioeconomic deprivation was derived by linking study member postcode with the index of multiple deprivation (HSE: 2001-6; SHS: 1995, 1998, 2003); serum C reactive protein, a marker of systemic inflammation, was measured from blood samples drawn by a nurse at a second home visit ${ }^{27}$ (HSE: 1998, 2003-6; SHS: 1998, 2003); physical activity was self reported (HSE: 1994, 1997-99, 2003, 2004, 2006, 2008; SHS: 1995, 1998, 2003) ${ }^{28}$; and from data on quantity of weekly alcohol intake in surveys (HSE: 1994-95, 1997-98, 1999-2000, 2001; SHS: 2003) ${ }^{29}$ we categorised study members into harmful drinkers ( $>14$ units/week ${ }^{30}$ ).

\section{Outcome ascertainment: cancer mortality and incidence}

Study members were linked to the National Health Service (NHS) central registries at Southport and Dumfries, UK, the procedures of which provide the vital status of study members and, when applicable, causes of death, which included cancer. Cancers deaths were denoted by cancer recorded as the underlying cause of death on the death certificate (as opposed to contributing cause). Cancer registrations for a diagnosis of a non-fatal malignancy (incidence) were also available for the three Scottish studies through the Scottish cancer registry. All cancers combined were denoted by ICD-9 (international 
classification of disease, ninth edition) $)^{31}$ codes 140-239, and ICD-10 ${ }^{32}$ codes C00-D48. Individual malignancies were categorised as follows (ordered by ICD-9 code): oesophagus (ICD-9 code 150, ICD-10 code C15), stomach (ICD-9 code 151, ICD-10 code C16), colorectal (ICD-9 codes 153-154, ICD-10 codes C18-C20), liver (ICD-9 code 155 , ICD-10 code C22), pancreas (ICD-9 code 157, ICD-10 code C25), lung (ICD-9 codes 162, ICD-10 codes C34), mesothelioma (ICD-9 codes 163, ICD-10 code C45), breast (female) (ICD-9 code 174, ICD-10 code C50), ovary (women) (ICD-9 code 183, ICD-10 code C56), prostate (men) (ICD-9 code 185, ICD-10 code C61), bladder (ICD-9 code 188, ICD-10 code C67), kidney (ICD-9 code 189, ICD10 codes $\mathrm{C64}$ and C65), central nervous system (ICD-9 code 191 and 192, ICD-10 codes C70-C72), non-Hodgkin's lymphoma (ICD-9 codes 200 and 202, ICD-10 codes C82C86), multiple myeloma (ICD-9 code 203, ICD-10 code C90.0), and leukaemia (ICD-9 codes 204-208, ICD-10 codes C91-C95). A category of cancer related to smoking was based on current evidence. ${ }^{33} 34$

\section{Patient involvement}

No patients were involved in setting the present research question nor the outcome measures, nor were they involved in developing plans for recruitment, design, or implementation of the study. No patients were asked to advise on interpretation or writing up of results. There are no plans to disseminate the results of the research to study participants or the relevant patient community.

\section{Statistical analyses}

We used raw data for all study years, with the exception of 1996 and 2007, when a psychological distress scale was not administered. In preliminary analyses, we were able to determine that the proportional hazards assumption had not been violated by inspecting the survival curves according to distress categories. With there also being no evidence of an interaction by sex for the association between psychological distress and cancer $(\mathrm{P}=0.63)$, we pooled data for men and women and adjusted the effect estimates for sex. Cox proportional hazards models ${ }^{35}$ were used to compute study specific hazard ratios with accompanying 95\% confidence intervals for the association between distress and each cancer mortality outcome. We used calendar time (months) as the time scale, with survivors having a censoring date of 15 February 2011. Hazard ratios were minimally adjusted (age and sex only) and maximally adjusted (age, sex, BMI, educational attainment, smoking status, and frequency of alcohol consumption). In the main analyses, we omitted cancer endpoints with too few cases $(<50)$ to provide stable effect estimates.

Subgroup analyses were conducted with data that were available only in selected surveys. Here, age and sex adjusted hazard ratios for the association between distress and cancer were additionally adjusted for physical activity, ${ }^{36} \mathrm{C}$ reactive protein, ${ }^{37}$ area level deprivation, ${ }^{38}$ and harmful levels of alcohol intake ${ }^{39}$ in turn, all of which have been linked with selected malignancies featured in the present analyses. We also constructed models using cancer incidence (for SHS only) to compare with results for distress and mortality in this group of studies.

We used the $\mathrm{I}^{2}$ statistic as a measure of the degree of inconsistency of effect estimate (heterogeneity) across studies (and cancer outcomes). Although preliminary analyses showed that the $\mathrm{I}^{2}$ statistic between studies varied between $0 \%$ and $38 \%$ depending on the cancer mortality outcome under investigation, we pooled the study specific effect estimates and their standard errors in random effects meta-analyses to provide conservative effect estimates. All analyses were computed with $R$ version 3.2.2, with the exception of data imputation, which was performed with SPSS (version 22).

\section{Results}

Tables 1 and 2 show the characteristics of study members according to each of the 16 included studies. Individual study sample size ranged from 7405 to 14573 people; there was no difference in mean psychological distress score across the studies.

Figure 1 shows the flow of participants from study induction through to analytical sample. About $18 \%$ $(n=36141)$ of study members were excluded between recruitment and analyses, largely because of refusal to be linked to death or cancer registration records. Individuals with an extant cancer diagnosis at baseline $(n=3875)$ were also excluded. This resulted in a maximum analytic sample of 163363 (55\% women, mean age 46.3 (range 16-102)).

Table 3 compares the characteristics of the analytical sample with study members who had been excluded. In general, absolute differences were small, though significance at conventional levels was common because of the high numbers of people in the analyses. On this basis, there seemed to be little evidence of selection bias. In the analytical sample, participants were around middle age at study induction (mean age 46.3; range 16-102); around half were women (54.9\%), and about a quarter (26.3\%) were smokers. Around two thirds of the sample left school after the mandatory age.

Based on the 163363 study members in the sample for analysis, we examined baseline covariates according to the four categories of psychological distress (table 4). As anticipated, study members with higher distress scores had less favourable levels of a range of characteristics, some of which are known risk factors for selected cancers. Thus, relative to people with lower distress levels, the more distressed study members were more likely to have a basic education, smoke, and be obese. The only exception to this observation was the weekly intake of alcohol beverages, which was lower in people reporting higher levels of distress.

During a mean (SD) follow-up of 9.5 (4.3) years across the 16 studies there were 16267 deaths, 4353 of which were ascribed to cancer of any site. Figure 2 shows the age and sex adjusted relation between psychological distress and mortality from all cancer sites combined according to each of the 16 studies featured in the present meta-analysis. With the exception of three studies with among the lowest number of cancer deaths (1997, 


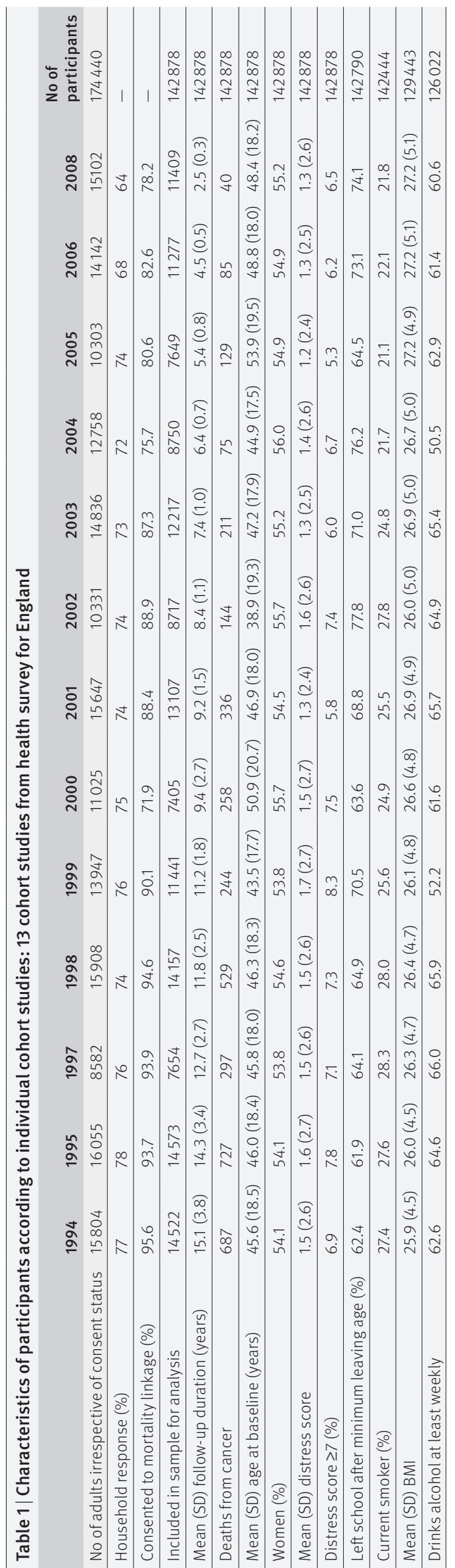

2005, and 2006 HSE), relative to individuals reporting lower distress scores (0-6), those with higher levels (7-12) experienced increased rates of total cancer mortality, though confidence intervals for all but five studies included unity. An $\mathrm{I}^{2}$ statistic of $2 \%$ suggests essentially no statistical heterogeneity in the study specific estimates. In the 16 studies in aggregate, after adjustment for age and sex, higher levels of distress were associated with a 32\% greater risk of total cancer mortality (hazard ratio 1.32, 95\% confidence interval 1.18 to 1.48$)$.

Figure 3 shows analyses for distress according to 16 independent (non-overlapping) cancer presentations plus some of these sites in aggregate (total cancer and cancers related and not related to smoking). For all the malignancy endpoints featured in analyses in which hazard ratios were adjusted for age and sex, higher death rates were apparent in people with higher levels of distress, though significance at conventional levels was not always apparent. Thus, of the individual sites, in the model with age and sex, the weakest effects were seen for lung cancer and the strongest for mesothelioma. Some of these estimates were imprecise, as evidenced by the wide confidence intervals because of a low number of cancer deaths. We also show the impact of adjustment for a range of further covariates. Relative to the age and sex adjusted hazard ratios, adjustment for covariates that included socioeconomic position (education) and health behaviours (cigarette smoking, alcohol intake) had little attenuating effect; indeed, in some cases, positive confounding was apparent. One exception was tobacco related cancer (including lung), for which, unsurprisingly, the addition of smoking to the multivariable model led to partial attenuation of the association with distress (table $\mathrm{A}$ in appendix 1 shows the impact of control for individual confounding factors in the multivariable model).

Table 5 shows the four categories of psychological distress we used to explore dose-response associations with different presentations of cancer. In these analyses there was some evidence of stepwise effects across the full distress range for cancer of the colorectum and prostate. With different models being based on different analytical samples because of missing covariates, we recomputed these effects estimates in a non-missing dataset-that is, the same sample size in both modelsand our results were unchanged.

We then carried out some planned subgroup analyses. Firstly, as described, certain potential covariates (physical activity, $\mathrm{C}$ reactive protein, area based deprivation, quantity of alcohol consumed) were collected only in selected studies and therefore did not feature in our main analyses. Because of the smaller numbers, we were able to examine the impact of adjustment for these covariates only on the relation between distress and all cancers combined (table B in appendix 1). The strength of the relation between distress and total cancer was little changed. Secondly, to explore the role of reverse causality-people entering the studies might have some symptoms of undiagnosed cancer, including pain and tiredness, which could cause, or be taken for, mental 
Table 2 | Characteristics of participants according to individual cohort studies: three cohort studies from Scottish health survey

\begin{tabular}{lllll} 
& 1995 & 1998 & 2003 & $\begin{array}{l}\text { No of } \\
\text { participants }\end{array}$ \\
No of adults irrespective of consent status & 7932 & 9040 & 8092 & 25064 \\
\hline Household response & - & 77 & 67 & - \\
\hline Consented to mortality linkage & 85.3 & 86.9 & 87.9 & - \\
\hline Included in analytic sample & 6484 & 7532 & 6469 & 20485 \\
\hline Mean (SD) follow-up duration (years) & $13.8(2.0)$ & $10.7(1.8)$ & $5.7(0.8)$ & 20485 \\
\hline Deaths from cancer & 194 & 281 & 116 & 20485 \\
\hline Cancer incidence & 484 & 590 & 292 & 20485 \\
\hline Mean (SD) age at baseline (years) & $40.5(13.2)$ & $45.3(15.6)$ & $49.0(17.3)$ & 20485 \\
\hline Women (\%) & 55.0 & 55.9 & 56.1 & 20485 \\
\hline Mean (SD) distress score & $1.7(2.8)$ & $1.6(2.8)$ & $1.4(2.8)$ & 20485 \\
\hline Distress score $\geq 7$ & 8.5 & 8.5 & 7.9 & 20485 \\
\hline Left school after minimum leaving age (\%) & 66.0 & 62.6 & 65.7 & 20468 \\
\hline Current smoker (\%) & 37.3 & 34.9 & 26.7 & 20356 \\
\hline Mean (SD) BMl & $26.1(4.6)$ & $26.7(4.9)$ & $27.3(5.1)$ & 18379 \\
\hline Drinks alcohol at least weekly & 63.0 & 61.0 & 60.7 & 18227 \\
\hline
\end{tabular}

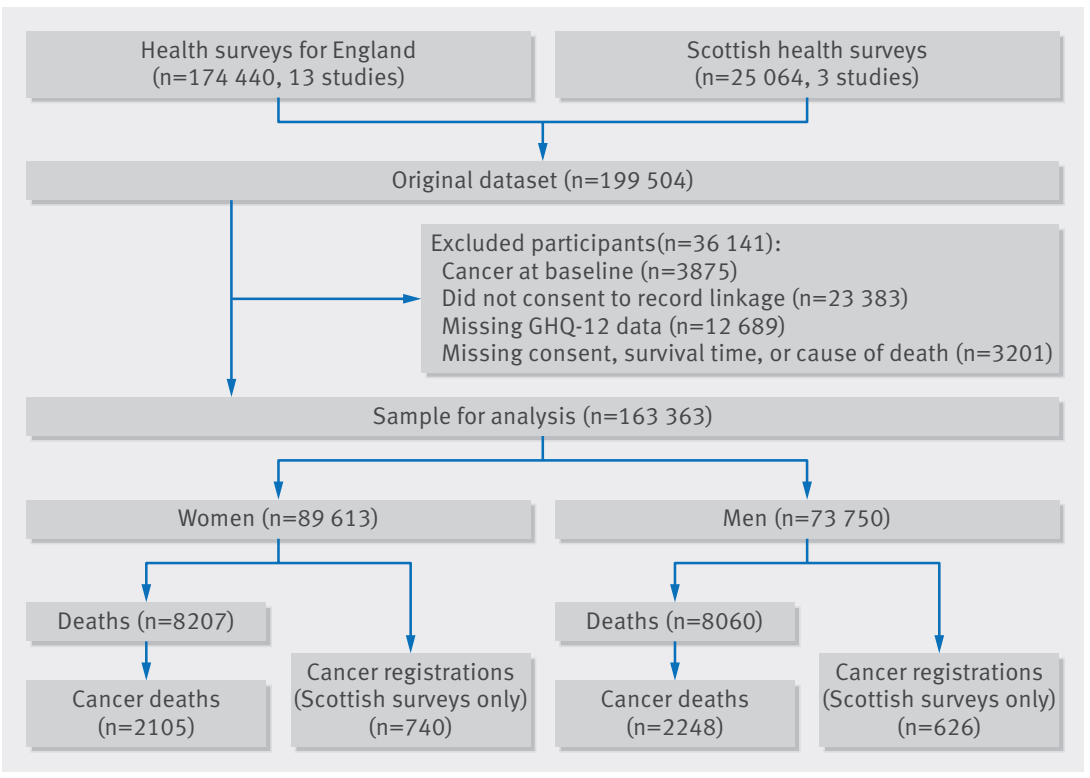

Fig 1 Study members from induction through to sample for analysis: follow-up of 16 cohort studies from health survey for England and Scottish health survey $(n=163363)$. People excluded can fall into more than one category so total exceeds 36141

distress-we excluded study members who died in the first five years of follow-up from the particular endpoint featured in each analysis. In doing so, we found that most of the associations between distress and cancer were largely unaffected (fig A in appendix 2).

Table 3 | Baseline characteristics of survey participants included and excluded from analyses: 16 cohort studies from health survey for England and Scottish health survey

\begin{tabular}{llll} 
& Included & Excluded & P value \\
No of study members & 163363 & 36141 & - \\
\hline Mean (SD) distress score & $1.5(2.6)$ & $1.5(2.7)$ & 0.12 \\
\hline Mean (SD) age (years) & $46.3(18.3)$ & $51.9(21.4)$ & $<0.001$ \\
\hline Women (\%) & 54.9 & 58.2 & $<0.001$ \\
\hline Mean (SD) BMI & $26.6(4.8)$ & $26.5(4.9)$ & 0.009 \\
\hline Left school after minimum leaving age (\%) & 67.9 & 61.1 & $<0.001$ \\
\hline Current smoker (\%) & 26.3 & 26.1 & 0.09 \\
\hline Drinks alcohol at least weekly (\%) & 62.0 & 49.1 & $<0.001$ \\
\hline
\end{tabular}

Thirdly, in related analyses, given that death data combine both incidence and survival, we examined if there was a relation between distress and incidence based on registration of a cancer diagnosis (data available only for the three Scottish studies) as this is more proximal to the exposure of interest and therefore the analyses potentially provide greater insights into aetiology. Figure 4 shows that there was some evidence of differential effects for cancer of all locations combined and colorectal cancer, such that the associations between distress and incidence were weaker, though the latter analysis particularly was compromised by relatively few events.

Lastly, 18\% ( $n=36141)$ of study members were excluded between recruitment and analyses, largely because of refusal to be linked to death or cancer registration records (fig 1). Accordingly, for each of the 16 cohort studies in the analysis we used multiple multivariate imputation based on baseline variables available for any missing values. We ran five cycles of regression, which generated five imputation datasets for each of the 16 studies, and the results were obtained by averaging results across each of these five datasets using the approach of Rubin. ${ }^{40}$ This procedure takes into account the uncertainty in the imputation process as well as uncertainty from random variation. Meta-analysis of the results from these imputed study specific data (table $C$ in appendix 1 ) gave similar results to those seen with the non-missing dataset as reported in the present paper.

\section{Discussion \\ Principal findings}

In this pooling of unpublished individual participant data, we found that people in the highest distress grouping relative to the lowest experienced increased rates of death from selected cancers. Thus, after adjustment for covariates that are known risk factors for selected malignancies, such as adverse health behaviours, and with reverse causality (by left censoring) and missing data (by imputation) taken into consideration, the most consistently robust effects were evident for carcinoma of the colorectum, prostate, pancreas, and oesophagus and for leukaemia. For two of these malignancies-colorectal and prostate-a gradient was apparent, such that the greater level of distress, the higher the risk of cancer mortality. These associations provide partial support for our hypotheses based on plausible mechanisms of effect.

\section{Usefulness of the present study}

Our findings could be important in advancing understanding of the role of psychological distress in cancer aetiology and cancer progression as investigators attempt to ascertain what role this and other psychological factors (such as psychosocial stress, cognition, personality, life satisfaction) have, if any, in prevention and prognosis. By contextualising the predictive value of distress for risk of cancer by comparing it with established non-psychological risk factors using data from the present study (table D in appendix 1), it is evident 


\begin{tabular}{|c|c|c|c|c|c|}
\hline & \multicolumn{4}{|c|}{ Distress score ${ }^{\star}$} & \multirow[b]{2}{*}{ P value } \\
\hline & 0 & $1-3$ & $4-6$ & $7-12$ & \\
\hline No of people & 97273 & 41466 & 13114 & 11510 & - \\
\hline Mean (SD) age (years) & $47.0(18.0)$ & $45.3(19.0)$ & $44.9(18.8)$ & $46.6(17.4)$ & $<0.001$ \\
\hline Female (\%) & 52.0 & 56.8 & 62.4 & 63.5 & $<0.001$ \\
\hline Obese (\%) & 20.4 & 20.5 & 21.1 & 23.7 & $<0.001$ \\
\hline Left school after minimum leaving age (\%) & 68.7 & 68.2 & 66.7 & 62.1 & $<0.001$ \\
\hline Current smoker (\%) & 24.0 & 27.1 & 31.0 & 37.2 & $<0.001$ \\
\hline Drinks alcohol at least weekly (\%) & 63.9 & 61.4 & 57.7 & 53.8 & $<0.001$ \\
\hline
\end{tabular}

*Higher score indicates greater degree of psychological distress

that, aside from lung cancer for which cigarette smoking has a known causal influence, the hazard ratios for higher levels of distress are of similar magnitude to those for current smoking and obesity for selected cancer presentations. Individually, however, none of these risk factors is powerful enough to determine a person's risk: in analyses of death from a common cancer such as colorectal for instance, the sensitivity-the proportion of people who went on to develop a disease who also had the risk factor at baseline-was only $8 \%$ for psychological distress, $17 \%$ for current cigarette smoking, and $27 \%$ for obesity. As has been shown for cardiovascular disease, however, where multifactorial algorithms are in widespread use in general practice (such as Framingham, ${ }^{41}$ QRisk $^{42}$ ), collectively, these and other risk factors might have predictive utility for common cancer presentations (such as colorectal, breast, prostate). In developing such algorithms, psychological distress could be considered as a component, which is not currently the case. ${ }^{43}$ That these risk

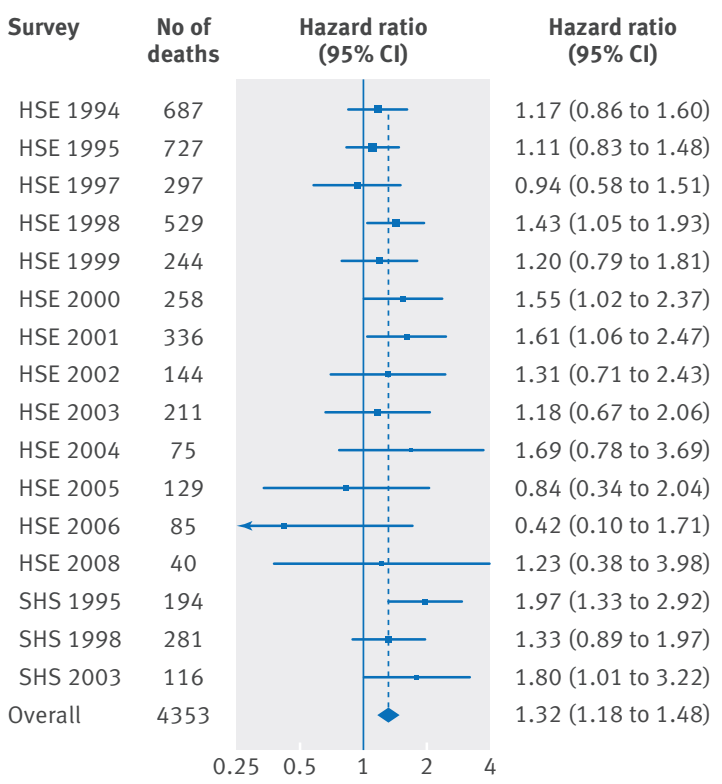

Fig 2 | Hazard ratios (95\% confidence intervals) for psychological distress in relation to mortality from all cancers combined according to study: follow-up of 16 cohort studies from health survey for England (HSE) and Scottish health survey (SHS) $(n=163363)$. Hazard ratios (adjusted for age and sex) are for psychological distress score of 7-12 (most distressed) relative to $0-6.1^{2}=2 \%$ factors collectively have predictive value for selected cancers, together with the well established observations that cancers rates differ systematically across time, ${ }^{44}$ location, ${ }^{45}$ and migration pattern, ${ }^{46}$ strongly suggest that the initiation of cancers is not a simple stochastic process reflecting the number of tissue specific stem cell divisions, ${ }^{47}$ as has been suggested. ${ }^{48}$

\section{Study strengths and limitations}

Our study has some strengths, including the use of unpublished raw data from similarly conducted studies in the general population; as such, our findings are not subject to publication bias and comparison across studies is straightforward. We also used a large and well characterised dataset relative to many other studies in this specialty. Our work is of course not without its limitations. The assessment of psychological distress with the GHQ-12 referenced the preceding four week period. A short bout of distress is unlikely to be of aetiological relevance for a disease like cancer, which has a long induction period. There is evidence, however, that rates of recurrence are high for psychological distress. For instance, in a population of 4363 people in a similar age range to the present study members, we found that, based on the general health questionnaire (30 items) over a maximum of 19 years of surveillance (four phases of data collection), two thirds of the sample classified as distressed at baseline were also distressed on one or more occasion during follow-up. This is broadly consistent with findings for clinical depression. ${ }^{49}$ Thus, a single administration of a distress inventory seems to capture cases of long term depression and anxiety. This notwithstanding, having serial measurement of our exposure would have provided further insights into the chronicity of psychological distress and would have the added advantage of allowing us to mimic a trial in an observational context by identifying a group whose depression resolved over time and observing the occurrence of cancer in this group.

While we chose to include an array of cancer outcomes to explore specificity of association, not all of these were hypothesis driven. It is also the case that, given that our meta-analysis is based on observational studies, as well characterised as these studies were, confounding by known or unknown factors remains a perennial concern. The assessment of dietary characteristics, for example, was an omission. This problem could theoretically be circumvented in a randomised controlled trial of people undergoing treatment for depression and anxiety who are also subject to surveillance for cancer events where, if it is genuinely causal, a reversal of symptoms of distress would produce a lower rate of cancer in treated patients. While such an aetiological trial has been conducted in the context of cardiovascular disease- reduction in depression produced a lower risk of total mortality in one $\mathrm{e}^{50}$ but had no impact on myocardial reinfarction rates in another ${ }^{51}$ the logistics involved with the size and duration of a trial for multiple cancer presentations are likely to be prohibitive. An alternative but related approach would be to use Mendelian randomisation in the context of 


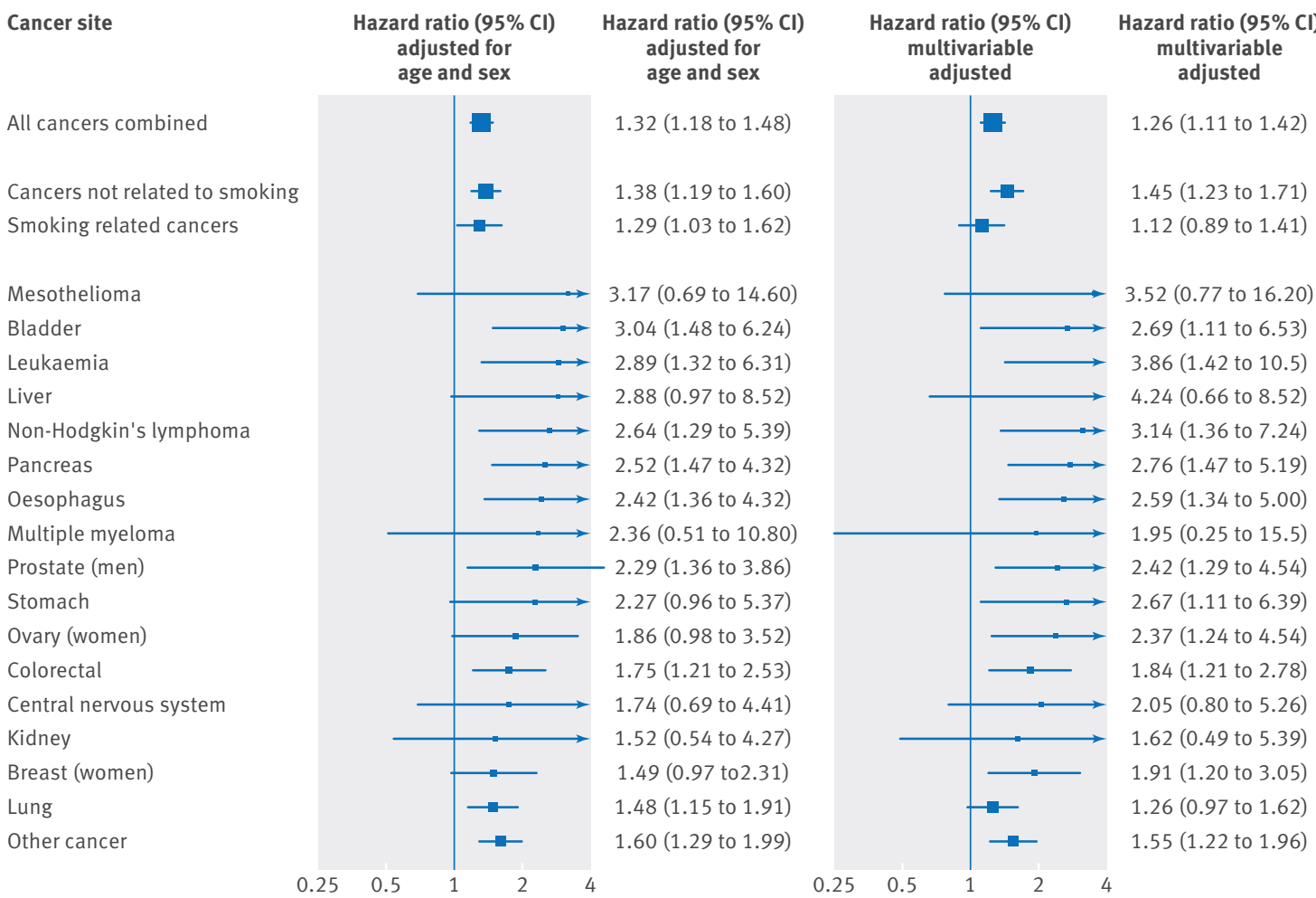

Fig 3 | Hazard ratios (95\% confidence interval) for psychological distress in relation to selected cancer death outcomes: follow-up of 16 cohort studies from health survey for England and Scottish health survey $(n=163363)$. Hazard ratios are for psychological distress score of 7-12 (most distressed) relative to 0-6, and are age and-except in single sex analyses-sex adjusted $\left(I^{2}=15 \%\right)$, or multivariable adjusted (age, sex, BMI, educational attainment, smoking status, and alcohol consumption; $\left.\left.\right|^{2}=37 \%\right)$

observational data in which a gene variant for psychological distress in principle provides an unconfounded estimate of the relation between an exposure and a disease outcome. ${ }^{52}$ As the genes for depression get identified, ${ }^{53}$ this represents a realistic proposition; unfortunately, the studies that comprise the present collaboration did not capture genetic material.

\section{Comparison with other studies}

The present analyses considerably extend our existing work, ${ }^{21}$ where we have shown that higher levels of distress were related to major causes of mortality, including cardiovascular disease, external causes of death, and all cancers combined, by exploring the link between distress and 16 different cancer presentations. We are not aware of previous meta-analyses on symptoms of psychological distress in relation to site specific cancer. In a recent meta-analysis of the occurrence of cancer subsequent to the assessment of major depression, ${ }^{13}$ which used study rather than individual level data and excluded studies in which investigators captured depressive symptoms, the aggregated result for depression and cancer for all malignancies across the 13 studies included unity (relative risk 1.12, 95\% confidence interval 0.99 to 1.26 ). This was of markedly lower magnitude than our estimate between psychological distress and overall cancer (multivariable adjusted hazard ratio $1.32,95 \%$ confidence interval 1.18 to 1.48 ). The discordant findings of prospective studies published since that meta-analysis have not clarified matters. ${ }^{54-60}$ Even within the same study, all cancers combined showed opposing gradients with depression in sex stratified analyses. ${ }^{59}$ Studies of sufficient scale to explore site specific associations with depression are rare, and the few that have been conducted show null effects for colon, lung, and prostate. ${ }^{57} 60$

\section{Mechanisms of effect}

Cancer mortality according to anatomical site-the main outcome in our study-is a composite of the onset of cancer (aetiology) together with survival from the disease (prognosis). The influence of psychological distress on processes acting at either or both of these disease stages could therefore influence the risk of cancer mortality. Moreover, these mechanisms can be direct (biological) and/or indirect (behavioural), and their action cancer specific and/or common to multiple presentations.

People with chronic distress typically have a less favourable lifestyle relative to those with lower levels, ${ }^{12}$ and this has been advanced as one means by which distress can be embodied, so increasing the risk of cancer. While we controlled for cigarette smoking, heavy alcohol intake, and physical inactivity in our analyses-and the associations held-health seeking behaviours might also be important, perhaps at a later stage in the disease process. Thus, people who are distressed might be less likely to comply with requests for screening, ${ }^{61}$ 


\begin{tabular}{|c|c|c|c|c|c|c|c|c|}
\hline \multirow[b]{2}{*}{ Cancer and adjustment } & \multirow[b]{2}{*}{ Deaths ${ }^{*}$} & \multirow[b]{2}{*}{ No of people } & \multicolumn{4}{|c|}{ Distress score } & \multirow{2}{*}{$\begin{array}{l}\text { P value } \\
\text { for trend }\end{array}$} & \multirow{2}{*}{$\begin{array}{l}\text { Distress score } \\
(7-12 \text { v } 0-6)\end{array}$} \\
\hline & & & 0 & $1-3$ & 4-6 & 7-12 & & \\
\hline \multicolumn{9}{|l|}{ All cancers } \\
\hline Age and sex & 4353 & 163363 & 1 (ref) & 0.99 (0.92 to 1.08$)$ & 1.05 (0.91 to 1.20$)$ & $1.31(1.16$ to 1.49$)$ & $<0.001$ & $1.32(1.18$ to 1.48$)$ \\
\hline Multivariable $^{\dagger}$ & 3800 & 146008 & 1 & 0.95 (0.88 to 1.03$)$ & 0.93 (0.78 to 1.10$)$ & 1.22 (1.07 to 1.38$)$ & 0.04 & 1.26 (1.11 to 1.42$)$ \\
\hline \multicolumn{9}{|l|}{ Not related to smoking } \\
\hline Age and sex & 2323 & 163363 & 1 & 0.96 (0.87 to 1.06$)$ & 1.06 (0.89 to 1.27$)$ & 1.36 (1.17 to 1.59$)$ & $<0.001$ & 1.38 (1.19 to 1.60$)$ \\
\hline Multivariable & 2036 & 146008 & 1 & 0.94 (0.84 to 1.04$)$ & 1.01 (0.82 to 1.25$)$ & 1.43 (1.21 to 1.69$)$ & $<0.001$ & 1.45 (1.23 to 1.71$)$ \\
\hline \multicolumn{9}{|l|}{ Smoking related } \\
\hline Age and sex & 2030 & 163363 & 1 & 1.03 (0.93 to 1.15$)$ & 1.09 (0.86 to 1.38$)$ & $1.29(1.02$ to 1.64$)$ & 0.03 & 1.29 (1.03 to 1.62$)$ \\
\hline Multivariable & 1764 & 146008 & 1 & 0.98 (0.88 to 1.09$)$ & 0.93 (0.76 to 1.14$)$ & 1.09 (0.86 to 1.38$)$ & 0.92 & 1.12 (0.89 to 1.41$)$ \\
\hline \multicolumn{9}{|l|}{ Lung } \\
\hline Age and sex & 992 & 163363 & 1 & 1.10 (0.94 to 1.28$)$ & 1.45 (1.05 to 2.00$)$ & 1.55 (1.17 to 2.06$)$ & $<0.001$ & 1.48 (1.15 to 1.91$)$ \\
\hline Multivariable & 865 & 146008 & 1 & 1.04 (0.88 to 1.22$)$ & 1.21 (0.90 to 1.64$)$ & $1.26(0.95$ to 1.66$)$ & 0.13 & 1.26 (0.97 to 1.62$)$ \\
\hline \multicolumn{9}{|l|}{ Colorectal } \\
\hline Age and sex & 391 & 163363 & 1 & 1.09 (0.86 to 1.39) & 1.48 (1.02 to 2.14$)$ & $1.77(1.20$ to 2.59$)$ & 0.01 & 1.75 (1.21 to 2.53$)$ \\
\hline Multivariable & 341 & 135888 & 1 & 1.11 (0.86 to 1.44$)$ & 1.56 (1.05 to 2.33$)$ & 1.89 (1.23 to 2.90$)$ & 0.01 & 1.84 (1.21 to 2.78$)$ \\
\hline \multicolumn{9}{|l|}{ Breast (female) } \\
\hline Age & 257 & 89613 & 1 & 0.93 (0.63 to 1.37$)$ & 0.74 (0.38 to 1.44$)$ & 1.36 (0.87 to 2.13$)$ & 0.97 & 1.49 (0.97 to 2.31) \\
\hline Multivariable & 213 & 78707 & 1 & 1.03 (0.66 to 1.60$)$ & 0.97 (0.47 to 1.99$)$ & 1.69 (1.04 to 2.73$)$ & 0.41 & 1.91 (1.20 to 3.05$)$ \\
\hline \multicolumn{9}{|l|}{ Prostate } \\
\hline Age & 253 & 73750 & 1 & 0.87 (0.62 to 1.22$)$ & 1.91 (1.21 to 3.01$)$ & 2.28 (1.33 to 3.92$)$ & 0.004 & 2.29 (1.36 to 3.86$)$ \\
\hline Multivariable & 216 & 67301 & 1 & 0.93 (0.64 to 1.35$)$ & 1.81 (0.93 to 3.52$)$ & $2.38(1.25$ to 4.57$)$ & 0.01 & 2.42 (1.29 to 4.54$)$ \\
\hline \multicolumn{9}{|l|}{ Pancreas } \\
\hline Age and sex & 244 & 163363 & 1 & 1.18 (0.87 to 1.59$)$ & 1.32 (0.75 to 2.33$)$ & 2.09 (1.19 to 3.65$)$ & 0.35 & 2.52 (1.47 to 4.32$)$ \\
\hline Multivariable & 218 & 146008 & 1 & 1.16 (0.84 to 1.59$)$ & $1.12(0.55$ to 2.26$)$ & 2.08 (1.11 to 3.89) & 0.772 & 2.76 (1.47 to 5.19$)$ \\
\hline Oesophagus & & & & & & & & \\
\hline Age and sex & 209 & 163363 & 1 & 1.09 (0.79 to 1.52$)$ & 1.13 (0.51 to 2.50$)$ & 2.43 (1.33 to 4.44$)$ & 0.314 & $2.42(1.36$ to 4.32$)$ \\
\hline Multivariable & 174 & 135888 & 1 & 1.02 (0.71 to 1.47$)$ & 1.34 (0.60 to 3.00$)$ & 2.48 (1.25 to 4.92$)$ & 0.38 & 2.59 (1.34 to 5.00$)$ \\
\hline Stomach & & & & & & & & \\
\hline Age and sex & 156 & 155714 & 1 & 0.89 (0.60 to 1.32$)$ & 0.83 (0.33 to 2.10$)$ & 2.22 (0.91 to 5.39) & 0.72 & 2.27 (0.96 to 5.37) \\
\hline Multivariable & 141 & 139323 & 1 & 0.90 (0.60 to 1.37$)$ & 0.84 (0.30 to 2.37$)$ & $2.52(1.03$ to 6.20$)$ & 0.97 & 2.67 (1.11 to 6.39$)$ \\
\hline Ovary & & & & & & & & \\
\hline Age & 125 & 83424 & 1 & 0.93 (0.59 to 1.47$)$ & 1.53 (0.76 to 3.07$)$ & 1.71 (0.88 to 3.32$)$ & 0.14 & 1.86 (0.98 to 3.52$)$ \\
\hline Multivariable & 111 & 67823 & 1 & 0.96 (0.60 to 1.55$)$ & 1.87 (0.81 to 4.27$)$ & 2.18 (1.11 to 4.28$)$ & 0.04 & 2.37 (1.24 to 4.54$)$ \\
\hline Bladder & & & & & & & & \\
\hline Age and sex & 121 & 163363 & 1 & 1.44 (0.93 to 2.22$)$ & $2.42(1.10$ to 5.31$)$ & 3.31 (1.55 to 7.09$)$ & 0.01 & 3.04 (1.48 to 6.24$)$ \\
\hline Multivariable & 107 & 146008 & 1 & 1.27 (0.79 to 2.05$)$ & 1.92 (0.81 to 4.57$)$ & 2.55 (1.02 to 6.35$)$ & 0.18 & 2.69 (1.11 to 6.53$)$ \\
\hline Non-Hodgkin's lymphom & & & & & & & & \\
\hline Age and sex & 110 & 151954 & 1 & 0.77 (0.44 to 1.37$)$ & 1.19 (0.46 to 3.10$)$ & 1.99 (0.97 to 4.10) & 0.46 & 2.64 (1.29 to 5.39) \\
\hline Multivariable & 99 & 135888 & 1 & $0.70(0.38$ to 1.27$)$ & $1.22(0.46$ to 3.24$)$ & $2.46(1.06$ to 5.73$)$ & 0.33 & 3.14 (1.36 to 7.24$)$ \\
\hline Central nervous system & & & & & & & & \\
\hline Age and sex & 105 & 154613 & 1 & 1.10 (0.67 to 1.81$)$ & 1.80 (0.83 to 3.91$)$ & 2.05 (0.78 to 5.41$)$ & 0.10 & 1.74 (0.69 to 4.41$)$ \\
\hline Multivariable & 96 & 138348 & 1 & 1.07 (0.63 to 1.83$)$ & $2.02(0.92$ to 4.42$)$ & $2.46(0.91$ to 6.63$)$ & 0.06 & $2.05(0.80$ to 5.26$)$ \\
\hline Leukaemia & & & & & & & & \\
\hline Age and sex & 101 & 163363 & 1 & 0.99 (0.60 to 1.66$)$ & 3.08 (1.16 to 8.15$)$ & 2.61 (1.06 to 6.44$)$ & 0.11 & 2.89 (1.32 to 6.31$)$ \\
\hline Multivariable & 87 & 135999 & 1 & 1.13 (0.65 to 1.97$)$ & $3.86(0.72$ to 20.8$)$ & $3.92(1.36$ to 11.2$)$ & 0.06 & $3.86(1.42$ to 10.5$)$ \\
\hline Kidney & & & & & & & & \\
\hline Age and sex & 90 & 163363 & 1 & 0.72 (0.39 to 1.34$)$ & 1.40 (0.54 to 3.66$)$ & 1.22 (0.43 to 3.45$)$ & 0.82 & 1.52 (0.54 to 4.27$)$ \\
\hline Multivariable & 79 & 127961 & 1 & 0.80 (0.43 to 1.51$)$ & $1.67(0.63$ to 4.40$)$ & $1.40(0.41$ to 4.77$)$ & 0.79 & 1.62 (0.49 to 5.39$)$ \\
\hline Liver & & & & & & & & \\
\hline Age and sex & 61 & 146997 & 1 & 2.24 (1.12 to 4.48$)$ & 3.67 (1.12 to 12.0$)$ & 3.09 (0.97 to 9.90) & 0.07 & 2.88 (0.97 to 8.52$)$ \\
\hline Multivariable & 53 & 125978 & 1 & 1.57 (0.86 to 2.85$)$ & NA & $1.73(0.53$ to 16.3$)$ & 0.20 & 4.24 (0.66 to 27.0$)$ \\
\hline Multiple myeloma & & & & & & & & \\
\hline Age and sex & 51 & 135555 & 1 & 0.60 (0.27 to 1.34$)$ & 2.07 (0.42 to 10.2$)$ & 1.80 (0.37 to 8.7$)$ & 0.72 & 2.36 (0.51 to 10.8$)$ \\
\hline Multivariable & 41 & 111534 & 1 & $0.70(0.25$ to 1.99$)$ & 4.79 (0.42 to 55.3$)$ & 1.41 (0.18 to 11.3$)$ & 0.83 & $1.95(0.25$ to 15.5$)$ \\
\hline Mesothelioma & & & & & & & & \\
\hline Age and sex & 51 & 134208 & 1 & 1.09 (0.49 to 2.41$)$ & 2.38 (0.27 to 20.7$)$ & 3.42 (0.68 to 17.1$)$ & 0.80 & 3.17 (0.69 to 14.6$)$ \\
\hline Multivariable & 51 & 120461 & 1 & $1.12(0.51$ to 2.50$)$ & 2.39 (0.27 to 21.6$)$ & 3.91 (0.78 to 19.6$)$ & 0.62 & $3.52(0.77$ to 16.2$)$ \\
\hline "Other" cancers & & & & & & & & \\
\hline Age and sex & 1036 & 163363 & 1 & 1.08 (0.93 to 1.25$)$ & 1.36 (1.09 to 1.70$)$ & 1.66 (1.33 to 2.08$)$ & $<0.001$ & 1.60 (1.29 to 1.99$)$ \\
\hline
\end{tabular}

\begin{tabular}{lllllllll}
\hline Age and sex & 1036 & 163363 & 1 & $1.08(0.93$ to 1.25$)$ & $1.36(1.09$ to 1.70$)$ & $1.66(1.33$ to 2.08$)$ & $<0.001$ & $1.60(1.29$ to 1.99$)$ \\
\hline Multivariable & 908 & 146008 & 1 & $1.01(0.86$ to 1.19$)$ & $1.30(1.01$ to 1.67$)$ & $1.58(1.24$ to 2.01$)$ & $<0.001$ & $1.55(1.22$ to 1.96$)$ \\
\hline
\end{tabular}

$\mathrm{NA}=$ too few cancer deaths to run analyses.

*No of deaths and total numbers in each model varies for sex specific cancers and when no participants died with particular cancers in individual survey years resulting in that whole cohort study being excluded from meta-analysis.

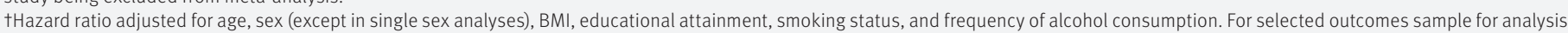
is markedly smaller than for all cancers combined because certain cohort studies, particularly those with shorter duration of follow-up, had not accumulated any deaths for endpoint of interest. 


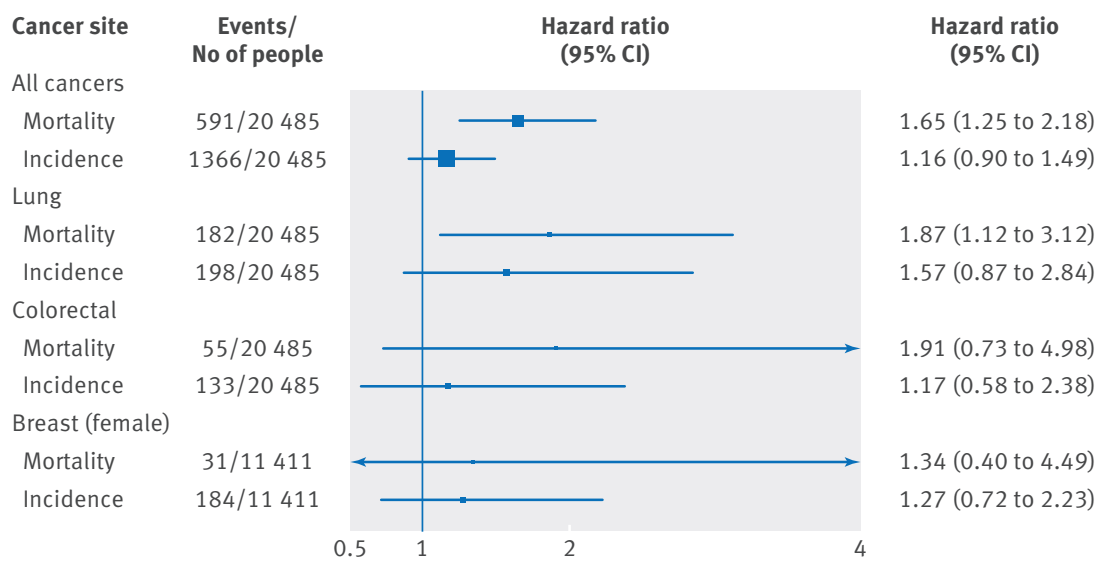

Fig 4 | Hazard ratios (95\% confidence interval) for psychological distress in relation to selected cancer outcomes: comparison of effects for incidence and mortality in follow-up of three cohort studies from Scottish health survey (SHS; $n=20485$ ). Hazard ratios (adjusted for age and sex) are for psychological distress score of 7-12 (most distressed) relative to $0-6$. Individuals with cancers registered before baseline $(n=696)$ were excluded from analyses of cancer incidence

resulting in a delayed diagnosis, and, once cancer is diagnosed, depression might hamper adherence to treatment. ${ }^{62}$ These findings, however, are notuniversal. ${ }^{63}$ We did not collect data on treatment behaviours in the present study.

Of the biological mechanisms, mood disorders such as depression have been implicated in immune pathways and are known to provoke inflammatory responses. Prolonged immune dysregulation can compromise the repair capacity of the exposed cells, potentially contributing to genetic instability and mutations, alterations in DNA repair, and inhibition of apoptosis. ${ }^{6465}$ Immune dysregulation can also lead to a worse prognosis for several carcinomas, including cancer of the colorectum, lung, mesothelium, and stomach. ${ }^{66}$ Depression and distress are also associated with markers of increased inflammation, such as interleukin 6, high sensitivity $C$ reactive protein, and soluble tumour necrosis factor receptor. ${ }^{67}$ In subgroup analyses, the associations between distress and cancer we observed were unchanged after the addition of circulating $\mathrm{C}$ reactive protein concentrations to the multivariable model, but we did not have data on a wider suite of inflammatory indicators. The lack of specificity of the relation between distress and cancer site in our analyses did not provide unambiguous insights into potential mechanisms, though, in general, the associations seemed stronger for some hormone related cancers, such as carcinoma of the prostate and ovaries. This observation accords with the notion of stress related mechanisms, which include dysregulation of the hypothalamic pituitary adrenal (HPA) ${ }^{68}$ and sympathetic adrenal medullary (SAM) axes. ${ }^{69}$

\section{Exploring the role of reverse causality}

It is plausible that the associations we found between distress and cancer reflect both the effects of cancerdiagnosed and undiagnosed-on mood, the effects of distress on cancer progression, or a combination. As described, it is well documented that a diagnosis of cancer can give rise to distress, ${ }^{70}$ and we dealt with this potential source of reverse causality by using the standard practice of excluding members with self reported malignancy at study entry. Having done so, when we explored the risk between distress and total cancer according to study, those with longer follow-up generally showed weaker associations. As duration of follow-up increases, the proportion of surviving people who had entered the study with unknown cancer diminishes relative to the total number of deaths from cancer; the influence of cancer on distress should likewise wane over time. Moreover, in analyses of the Scottish studies, the associations were somewhat weaker for cancer incidence than for cancer mortality, though these analyses were not well powered because of the lower number of new cancer cases. Taking these observations together, there was a suggestion that subclinical malignancy might have had an impact on mood. Thus, to explore the impact of occult cancer, we excluded study members who died in the first five years of follow-up. This practice is based on the assumption that people with occult cancers of the more lethal variety will have died during this period. In these analyses, the gradients between distress and cancer were, however, still seen.

In conclusion, our findings add to the growing evidence of an association between psychological distress and physical conditions by characterising new relations with death from selected cancer presentations. The extent to which these associations could be causal requires further testing with alternative study designs.

We thank Robert Miller for his advice and correction.

Contributors: GDB conceived and designed the study. ES was responsible for acquisition of data (including links to mortality and cancer registration (Scotland)). GDB, MK, and TCR produced an analytical plan. TCR was responsible for data analysis. TCR, MK, and GDB interpreted the results. GDB produced a first draft of the manuscript, and all authors provided intellectual input. TCR and GDB are guarantors.

Funding: This research received no specific grant from any funding agency in the public, commercial, or not-for-profit sectors. TCR is supported by Alzheimer Scotland through the Marjorie MacBeath bequest. MK is supported by the Medical Research Council (K013351) and NordForsk (the Nordic Programme on Health and Welfare). The views expressed herein by the authors are independent of all funding agencies.

Competing interests: All authors have completed the ICMJE uniform disclosure form at www.icmje.org/coi_disclosure.pdf and declare: no support from any organisation for the submitted work; no financial relationships with any organisations that might have an interest in the submitted work in the previous three years; and no other relationships or activities that could appear to have influenced the submitted work.

Ethical approval: The study was approved by the London research ethics council.

Data sharing: The baseline data for the surveys described herein are curated by the UK Data Archive at the University of Essex, and can be downloaded free of charge for non-commercial purposes from the Economic and Social Data Service (www.esds.ac.uk/findingData/ hseTitles.asp). Syntax for the present analyses are available from the authors.

Transparency: The lead author affirms that the manuscript is an honest, accurate, and transparent account of the study being reported; that no important aspects of the study have been omitted; and that any discrepancies from the study as planned have been explained.

This is an Open Access article distributed in accordance with the terms of the Creative Commons Attribution (CC BY 3.0) license, which permits others to distribute, remix, adapt and build upon this work, for commercial use, provided the original work is properly cited. See: http://creativecommons.org/licenses/by/3.0/. 
1 Mettler CC, Mettler FA. A history of medicine. Philidelphia: Blakiston; 1947.

2 Kivimäki M, Nyberg ST, Batty GD, et al. IPD-Work Consortium. Job strain as a risk factor for coronary heart disease: a collaborative meta-analysis of individual participant data. Lancet 2012;380:1491-7. doi:10.1016/S0140-6736(12)60994-5.

3 Batty GD, Deary IJ, Zaninotto P. Association of Cognitive Function With Cause-Specific Mortality in Middle and Older Age: Follow-up of Participants in the English Longitudinal Study of Ageing. Am J Epidemiol 2016;183:183-90. doi:10.1093/aje/kwv139.

4 Jokela M, Pulkki-Råback L, Elovainio M, Kivimäki M. Personality traits as risk factors for stroke and coronary heart disease mortality: pooled analysis of three cohort studies. J Behav Med 2014;37:881-9. doi:10.1007/s10865-013-9548-z

5 Steptoe A, Kivimäki M. Stress and cardiovascular disease. Nat Rev Cardiol 2012;9:360-70. doi:10.1038/nrcardio.2012.45.

6 Nicholson A, Kuper H, Hemingway H. Depression as an aetiologic and prognostic factor in coronary heart disease: a meta-analysis of 6362 events among 146538 participants in 54 observational studies. Eur Heart J 2006;27:2763-74. doi:10.1093/eurhearti/ehl338.

7 Pan A, Sun Q, Okereke OI, Rexrode KM, Hu FB. Depression and risk of stroke morbidity and mortality: a meta-analysis and systematic review. JAMA 2011;306:1241-9. doi:10.1001/jama.2011.1282.

8 Batty GD, Russ TC, Stamatakis E, Kivimäki M. Psychological distress and risk of peripheral vascular disease, abdominal aortic aneurysm, and heart failure: pooling of sixteen cohort studies. Atherosclerosis 2014:236:385-8, doi:10.1016/j.atherosclerosis.2014.06.025.

9 Lozano R, Naghavi M, Foreman K, et al. Global and regional mortality from 235 causes of death for 20 age groups in 1990 and 2010: a systematic analysis for the Global Burden of Disease Study 2010. Lancet 2012;380:2095-128. doi:10.1016/S0140-6736(12)61728-0.

10 Kiecolt-Glaser JK, Robles TF, Heffner KL, Loving TJ, Glaser R. Psycho-oncology and cancer: psychoneuroimmunology and cancer. Ann Oncol 2002;13(Suppl 4):165-9. doi:10.1093/annonc/mdf655.

11 Spiegel D, Giese-Davis J. Depression and cancer: mechanisms and disease progression. Biol Psychiatry 2003;54:269-82. doi:10.1016/ S0006-3223(03)00566-3.

12 Pan A, Okereke OI, Sun Q, et al. Depression and incident stroke in women. Stroke 2011;42:2770-5. doi:10.1161/ STROKEAHA.111.617043.

13 Oerlemans ME, van den Akker M, Schuurman AG, Kellen E, Buntinx F. A meta-analysis on depression and subsequent cancer risk. Clin Pract Epidemiol Ment Health 2007;3:29. doi:10.1186/1745-0179-3-29.

14 Lewington S, Clarke R, Qizilbash N, Peto R, Collins R. Prospective Studies Collaboration. Age-specific relevance of usual blood pressure to vascular mortality: a meta-analysis of individual data for one million adults in 61 prospective studies. Lancet 2002;360:1903-13. doi:10.1016/S0140-6736(02)11911-8.

15 Seshasai SR, Kaptoge S, Thompson A, et al. Emerging Risk Factors Collaboration. Diabetes mellitus, fasting glucose, and risk of cause-specific death. N Engl J Med 2011:364:829-41. doi:10.1056/NEJMoa1008862.

16 Mindell J, Biddulph JP, Hirani V, et al. Cohort profile: the health survey for England. Int J Epidemiol 2012;41:1585-93. doi:10.1093/ije/dyr199.

17 Gray L, Batty GD, Craig P, et al. Cohort profile: the Scottish health surveys cohort: linkage of study participants to routinely collected records for mortality, hospital discharge, cancer and offspring birth characteristics in three nationwide studies. Int J Epidemiol 2010:39:345-50 doi:10.1093/ije/dyp155.

18 Goldberg D. Manual of the General Health Questionnaire. NFER, 1978.

19 Russ TC, Stamatakis E, Hamer M, Starr JM, Kivimäki M, Batty GD. Association between psychological distress and mortality: individual participant pooled analysis of 10 prospective cohort studies. BMJ 2012;345:e4933. doi:10.1136/bmj.e4933.

20 Russ TC, Hamer M, Stamatakis E, Starr JM, Batty GD. Psychological distress as a risk factor for dementia death. Arch Intern Med 2011;171:1858-9. doi:10.1001/archinternmed.2011.521.

21 Russ TC, Kivimäki M, Morling JR, Starr JM, Stamatakis E, Batty GD. Association Between Psychological Distress and Liver Disease Mortality: A Meta-analysis of Individual Study Participants. Gastroenterology 2015;148:958-966.e4. doi:10.1053/j. gastro.2015.02.004

22 Goldberg DP, Gater R, Sartorius N, et al. The validity of two versions of the GHQ in the WHO study of mental illness in general health care. Psychol Med 1997;27:191-7. doi:10.1017/S0033291796004242.

23 Stansfeld SA, Sharp DS, Gallacher JE, Yarnell JW. A population survey of ischaemic heart disease and minor psychiatric disorder in men. Psychol Med 1992;22:939-49. doi:10.1017/S0033291700038514.

24 Parikh-Patel A, Allen M, Wright WE. California Teachers Study Steering Committee. Validation of self-reported cancers in the California Teachers Study. Am J Epidemiol 2003;157:539-45. doi:10.1093/aje/ kwg006.

25 Bergmann MM, Calle EE, Mervis CA, Miracle-McMahill HL, Thun MJ, Heath CW. Validity of self-reported cancers in a prospective cohort study in comparison with data from state cancer registries. Am J Epidemiol 1998;147:556-62. doi:10.1093/oxfordjournals.aje. a009487.
26 Physical status: the use and interpretation of anthropometry. Report of a WHO Expert Committee. World Health Organ Tech Rep Ser 1995:854:1-452.

27 Batty GD, Bell S, Stamatakis E, Kivimäki M. Association of Systemic Inflammation With Risk of Completed Suicide in the General Population. JAMA Psychiatry 2016;73:993-5. doi:10.1001/jamapsychiatry.2016.1805.

28 Stamatakis E, Hamer M, O'Donovan G, Batty GD, Kivimaki M. A non-exercise testing method for estimating cardiorespiratory fitness: associations with all-cause and cardiovascular mortality in a pooled analysis of eight population-based cohorts. Eur Heart / 2013;34:7508. doi:10.1093/eurheartj/ehs097.

29 Kengne AP, Czernichow S, Stamatakis E, Hamer M, Batty GD. Gamma-glutamyltransferase and risk of cardiovascular disease mortality in people with and without diabetes: pooling of three British Health Surveys. J Hepatol 2012;57:1083-9. doi:10.1016/j. jhep.2012.06.034.

30 UK Chief Medical Officers. UK ChiefMedical Officers' Alcohol Guidelines Review: Summary of Proposed New Guidelines. Department of Health, 2015.

31 Anon. Manual of the International Statistical Classification of Diseases, Injuries, and Causes of Death (ninth revision).WHO, 1977.

32 Anon. International Statistical Classification of Diseases and Related Health Problems (10th revision).WHO, 1992.

33 International Agency for Research on Cancer. Tobacco Smoking and Involuntary Smoking. IARC Monographs on the Evaluation of Carcinogenic Risk to Humans. Vol 38. IARC, 2002

34 Batty GD, Kivimaki M, Gray L, Davey Smith G, Marmot MG, Shipley MJ. Cigarette smoking and site-specific cancer mortality: testing uncertain associations using extended follow-up of the original Whitehall study. Ann Oncol 2008;19:996-1002. doi:10.1093/annonc/mdm578.

35 Cox DR. Regression models and life-tables. J R Stat Soc [Ser B] 1972;34:187-220.

36 Batty D, Thune I. Does physical activity prevent cancer? Evidence suggests protection against colon cancer and probably breast cancer[editorial]. BMJ 2000;321:1424-5. doi:10.1136/bmj.321.7274.1424.

37 Heikkilä K, Ebrahim S, Lawlor DA. A systematic review of the association between circulating concentrations of $\mathrm{C}$ reactive protein and cancer. J Epidemiol Community Health 2007;61:824-33. doi:10.1136/jech.2006.051292.

38 McCartney G, Russ TC, Walsh D, et al. Explaining the excess mortality in Scotland compared with England: pooling of 18 cohort studies. J Epidemiol Community Health 2015;69:20-7. doi:10.1136/ jech-2014-204185.

39 Pelucchi C, Tramacere I, Boffetta P. Negri E, La Vecchia C. Alcohol consumption and cancer risk. Nutr Cancer 2011;63:983-90. doi:10.1080/01635581.2011.596642.

40 Rubin D. Multiple imputation for nonresponse in surveys. Wiley, 1987doi:10.1002/9780470316696.

41 Kannel WB, McGee DL. Composite scoring--methods and predictive validity: insights from the Framingham Study. Health Serv Res 1987;22:499-535.

42 Hippisley-Cox J, Coupland C, Vinogradova Y, Robson J, May M, Brindle P. Derivation and validation of QRISK, a new cardiovascular disease risk score for the United Kingdom: prospective open cohort study. BMJ 2007:335:136. doi:10.1136/bmi.39261.471806.55

43 Colditz GA, Atwood KA, Emmons K, et al. Harvard report on cancer prevention volume 4: Harvard Cancer Risk Index. Risk Index Working Group, Harvard Center for Cancer Prevention. Cancer Causes Control 2000;11:477-88. doi:10.1023/A:1008984432272.

44 American Cancer Society. Cancer Facts and Figures (http://www.cancer. org/research/cancerfactsstatistics/cancerfactsfigures2016/. 2016.

45 Jemal A, Center MM, DeSantis C, Ward EM. Global patterns of cancer incidence and mortality rates and trends. Cancer Epidemiol Biomarkers Prev 2010;19:1893-907. doi:10.1158/1055-9965. EPI-10-0437.

46 Ziegler RG, Hoover RN, Pike MC, et al. Migration patterns and breast cancer risk in Asian-American women. J Natl Cancer Inst 1993;85:1819-27. doi:10.1093/jnci/85.22.1819.

47 Davey Smith G, Relton CL, Brennan P. Chance, choice and cause in cancer aetiology: individual and population perspectives. Int J Epidemiol 2016;45:605-13. doi:10.1093/ije/dyw224.

48 Tomasetti C, Vogelstein B. Cancer etiology. Variation in cancer risk among tissues can be explained by the number of stem cell divisions. Science 2015;347:78-81. doi:10.1126/science.1260825.

49 American Psychiatric Association. Diagnostic and Statistical Manual of Mental Disorders.4th ed. American Psychiatric Association, 2000.

50 Gallo JJ, Morales KH, Bogner HR, et al. Long term effect of depression care management on mortality in older adults: follow-up of cluster randomized clinical trial in primary care. BMJ 2013;346:f2570. doi:10.1136/bmj.f2570.

51 Berkman LF, Blumenthal J, Burg M, et al. Enhancing Recovery in Coronary Heart Disease Patients Investigators (ENRICHD). Effects of treating depression and low perceived social support on clinical events after myocardial infarction: the Enhancing Recovery in Coronary Heart Disease Patients (ENRICHD) Randomized Trial. JAMA 2003;289:3106-16. doi:10.1001/jama.289.23.3106. 
52 Davey Smith G, Ebrahim S. 'Mendelian randomization': can genetic epidemiology contribute to understanding environmental determinants of disease?/nt J Epidemiol 2003:32:1-22. doi:10.1093/ije/dyg070.

53 Okbay A, Baselmans BM, De Neve JE, et al. LifeLines Cohort Study. Genetic variants associated with subjective well-being, depressive symptoms, and neuroticism identified through genome-wide analyses. Nat Genet 2016;48:624-33. doi:10.1038/ng.3552.

54 Phillips AC, Batty GD, Gale CR, et al. Generalized anxiety disorder, major depressive disorder, and their comorbidity as predictors of all-cause and cardiovascular mortality: the Vietnam experience study. Psychosom Med 2009;71:395-403. doi:10.1097/ PSY.0b013e31819e6706

55 Goldacre MJ, Wotton CJ, Yeates D, Seagroatt V, Flint J. Cancer in people with depression or anxiety: record-linkage study. Soc Psychiatry Psychiatr Epidemiol 2007;42:683-9. doi:10.1007/s00127-007-0211-2.

56 Chen YH, Lin HC. Increased risk of cancer subsequent to severe depression--a nationwide population-based study. J Affect Disord 2011;131:200-6. doi:10.1016/j.jad.2010.12.006

57 Lemogne C, Consoli SM, Melchior M, et al. Depression and the risk of cancer: a 15-year follow-up study of the GAZEL cohort. Am I Epidemiol 2013;178:1712-20. doi:10.1093/aje/kwt217.

58 Hung YN, Yang SY, Huang MC, et al. Cancer incidence in people with affective disorder: nationwide cohort study in Taiwan, 1997-2010. Br J Psychiatry 2014:205:183-8. doi:10.1192/bjp.bp.114.144741.

59 Chang HY, Keyes KM, Mok Y, Jung KJ, Shin YJ, Jee SH. Depression as a risk factor for overall and hormone-related cancer: the Korean cancer prevention study. J Affect Disord 2015;173:1-8. doi:10.1016/j.jad.2014.10.064.

60 Gross AL, Gallo JJ, Eaton WW. Depression and cancer risk: 24 years of follow-up of the Baltimore Epidemiologic Catchment Area sample. Cancer Causes Control 2010:21:191-9. doi:10.1007/ s10552-009-9449-1.
61 Lerman C, Kash K, Stefanek M. Younger women at increased risk for breast cancer: perceived risk, psychological well-being, and surveillance behavior. / Natl Cancer Inst Monogr 1994:(16):171-6.

62 Pirl WF, Roth AJ. Diagnosis and treatment of depression in cancer patients. Oncology (Williston Park) 1999;13:1293-301, discussion 1301-2, 1305-6.

63 Chang CK, Hayes RD, Broadbent MT, et al. A cohort study on mental disorders, stage of cancer at diagnosis and subsequent survival. BM] Open 2014;4:e004295.

64 Fedeles BI, Freudenthal BD, Yau E, et al. Intrinsic mutagenic properties of 5-chlorocytosine: A mechanistic connection between chronic inflammation and cancer. Proc Natl Acad Sci U S A 2015;112:E457180. doi:10.1073/pnas.1507709112.

65 Chida Y, Hamer M, Wardle J, Steptoe A. Do stress-related psychosocial factors contribute to cancer incidence and survival? Nat Clin Pract Oncol 2008;5:466-75. doi:10.1038/ncponc1134.

66 Diakos Cl, Charles KA, McMillan DC, Clarke SJ. Cancer-related inflammation and treatment effectiveness. Lancet Oncol 2014;15:e493-503. doi:10.1016/S1470-2045(14)70263-3.

67 Haapakoski R, Ebmeier KP. Alenius H, Kivimäki M Innate and adaptive immunity in the development of depression: An update on current knowledge and technological advances. Prog Neuropsychopharmacol Biol Psychiatry 2016:66:63-72. doi:10.1016/j.pnpbp.2015.11.012.

68 McEwen BS. Physiology and neurobiology of stress and adaptation: central role of the brain. Physiol Rev 2007;87:873-904. doi:10.1152/ physrev.00041.2006.

69 Yang EV, Glaser R. Stress-induced immunomodulation and the implications for health. Int Immunopharmacol 2002;2:315-24. doi:10.1016/S1567-5769(01)00182-5.

70 Hotopf M, Chidgey J, Addington-Hall J, Ly KL. Depression in advanced disease: a systematic review Part 1. Prevalence and case finding. Palliat Med 2002;16:81-97. doi:10.1191/02169216302pm507oa. 